\title{
Capacity of MRC on Correlated Rician Fading Channels
}

\author{
Khairi Ashour Hamdi, Senior Member, IEEE
}

\begin{abstract}
A new exact explicit expression is derived for the ergodic capacity of maximal ratio combining (MRC) schemes over arbitrarily correlated Rician fading channels. This is used to study the effects of channel correlation on the ergodic capacity. Numerical results reveal that both the phase and the magnitude of correlation have an impact on the ergodic capacity of Rician fading channels. This is in contrast to correlated Rayleigh fading, where the phase of the correlation has no effect on the ergodic capacity. It is also observed that negatively correlated branches in Rician fading may lead to an increase in ergodic capacity beyond that obtained by uncorrelated branches.
\end{abstract}

Index Terms-Channel capacity, maximal ratio combining (MRC), Rician fading, correlated fading, wireless SIMO systems.

\section{INTRODUCTION}

D IVERSITY reception is increasingly becoming a primary technique for improving the performance of radio communication systems in multipath propagation environments. Therefore, the performance of diversity schemes has recently received a considerable research efforts (e.g. [1]-[15]).

Recent relevant research on the evaluation of ergodic capacity in correlated fading channels include [3]-[12]. Boche and Jorswieck [3] has analytically shown that, correlation in Rayleigh fading causes a loss in the ergodic capacity (compared to uncorrelated fading), and gave a simple expression for the capacity loss in a fully correlated diversity system. Closed-form expressions for the capacity in the special case of correlated Rayleigh fading are given in [4]-[6]. Capacity analysis in case of Rician fading are given in [7]-[12]. Zhang and Liu in [7] used Porteous' lemma to find a simple approximate expression for the ergodic capacity in correlated Rician fading channels. Taylor series expansions are used in [8] to obtain more accurate approximations for the ergodic capacity in terms of the moments of the combined channel gain. Laguerre-series expansions are used in [9], [11] for the distribution of the combined SNR. The accuracy of some other approximations for the ergodic capacity in Rician fading has recently been analyzed in [12].

On the other hand, recent advances on performance analysis of digital communication systems in fading channels has recognized the potential importance of moment generating functions (MGF), or Laplace transforms, as a powerful tool for simplifying the analysis of diversity communication systems. This has led to simple expressions to average bit and symbol

Paper approved by M. Chiani, the Editor for Wireless Communications of the IEEE Communications Society. Manuscript received September 15, 2006; revised May 15, 2007.

K. A. Hamdi is with the School of Electrical \& Electronic Engineering, The University of Manchester. Sackville street, PO Box 88, Manchester M60 1QD, United Kingdom (e-mail: k.hamdi@manchester.ac.uk).

Digital Object Identifier 10.1109/TCOMM.2008.060381. error rates for a wide variety of digital signaling schemes on fading channels, including multichannel reception with correlated diversity (e.g. [14]-[16]). Key to these developments was the transformations of the conditional error rate expressions into different equivalent forms in which the conditional variable appears only as an exponent. For instance, the following identity has been widely employed to simplify the error rate analysis of coherent communication systems in fading

$$
\operatorname{erfc} \sqrt{\mathrm{SNR}}=\frac{2}{\pi} \int_{0}^{\pi / 2} \exp \left(\frac{-\mathrm{SNR}}{\sin ^{2} \theta}\right) d \theta .
$$

In this letter, we show that it is possible to express the conditional capacity $\log (1+\mathrm{SNR})$ in a form similar to (1), in which SNR appears only as an exponent. This facilitates using the moment generating functions and leads to a new simple expression for the ergodic capacity in arbitrarily correlated Rician fading channels.

This letter is organized as follows. The problem is stated in Section II, and the proposed new solution is given in Section III. Some numerical examples are given in Section IV, and Section V concludes this letter.

\section{The PROBLEM}

The purpose of this letter is to derive a simple expression for computing the following average, which represents the ergodic (average) capacity of the MRC diversity system

$$
\mathcal{C}=\mathbb{E}\left[\log _{2}\left(1+\boldsymbol{g}^{\dagger} \boldsymbol{g}\right)\right]
$$

where $\mathbb{E}[$.$] is the expectation operator, \boldsymbol{g}$ is a $M \times 1$ complex random vector that represents the normalized complex channel gains, and the superscript $\dagger$ denotes Hermitian transposition. Here, the instantaneous signal-to-noise ratio (SNR) at the $m$ th channel is $\left|g_{m}\right|^{2}, m=1,2, . ., M$, whereas $\boldsymbol{g}^{\dagger} \boldsymbol{g}=$ $\sum_{m=1}^{M}\left|g_{m}\right|^{2}$ is the combined SNR.

In Rician fading channels, $\boldsymbol{g}$ is a complex Gaussian vector having a probability density function (pdf) given by

$$
f(\boldsymbol{g})=\frac{1}{\pi^{M} \operatorname{det}(\boldsymbol{\Lambda})} e^{-(\boldsymbol{g}-\mu)^{\dagger} \boldsymbol{\Lambda}^{-1}(\boldsymbol{g}-\mu)}
$$

where $\mu=\mathbb{E}[\boldsymbol{g}]$ is the mean vector and $\boldsymbol{\Lambda}=$ $\mathbb{E}\left[(\boldsymbol{g}-\mu)^{\dagger}(\boldsymbol{g}-\mu)\right]$ is the covariance matrix. The Rician factor for the $m$ th channel is equal to $\kappa_{m}=\frac{\left|\mu_{m}\right|^{2}}{\Lambda_{m, m}}$.

Direct evaluation of the average in (2),

$$
\mathcal{C}=\int_{\boldsymbol{g}} \log _{2}\left(1+\boldsymbol{g}^{\dagger} \boldsymbol{g}\right) \frac{1}{\pi^{M} \operatorname{det}(\boldsymbol{\Lambda})} e^{-(\boldsymbol{g}-\mu)^{\dagger} \boldsymbol{\Lambda}^{-1}(\boldsymbol{g}-\mu)} d \boldsymbol{g}
$$

where the integration is over the $M$ dimensional vector $\boldsymbol{g}$, requires a huge computational efforts. Other common methods use the pdf for the quadratic form $\boldsymbol{g}^{\dagger} \boldsymbol{g}$, instead. However, 
simple closed-form expressions for the pdf of the Gaussian quadratic forms are known only in some special cases. Otherwise, the cumulative probability distribution function of arbitrary Gaussian quadratic forms are expressed as infinite series with coefficients being determined recursively in terms of the eigenvalues of the covariance matrix (e.g., [1], [9], [11], [18]).

On the other hand, closed-form expressions for the MGFs (or Laplace transforms) of Gaussian quadratic forms are readily known for arbitrarily complex Gaussian vectors (e.g. [17], [18])

$$
\mathcal{M}(z)=\mathbb{E}\left[e^{-z \boldsymbol{g}^{\dagger} \boldsymbol{g}}\right]=\frac{1}{\operatorname{det}\left(\boldsymbol{I}_{M}+z \boldsymbol{\Lambda}\right)} e^{-\mu^{\dagger}\left[z^{-1} \boldsymbol{I}_{M}+\boldsymbol{\Lambda}\right]^{-1} \mu}
$$

where $\boldsymbol{I}_{M}$ is the $M \times M$ identity matrix.

This has been successfully employed in [14] and [15] to obtain simple expressions for bit and symbol error rates of different digital communication systems. However, in order to utilize (5) for computing the ergodic capacity, it is required, firstly, to represent the conditional capacity expression $\log _{2}\left(1+\boldsymbol{g}^{\dagger} \boldsymbol{g}\right)$ in a different equivalent form in which the quadratic random variable $\boldsymbol{g}^{\dagger} \boldsymbol{g}$ would appear only as an exponent. In next section, we show that this is possible.

\section{The ERgODIC CAPACITY EVALUATION}

In order to employ the available closed-form expression for the MGF of the Gaussian quadratic forms (5) for evaluating the ergodic capacity in correlated Rician fading, we need firstly the following lemma.

Lemma 1: For any $x>0$

$$
\ln (1+x)=\int_{0}^{\infty} \frac{1}{z}\left(1-e^{-x z}\right) e^{-z} d z .
$$

Proof: The proof is given in the Appendix.

Now, with (6), the conditional capacity expression $\log _{2}\left(1+\boldsymbol{g}^{\dagger} \boldsymbol{g}\right)$ can be expressed in the following desirable form

$$
\log _{2}\left(1+\boldsymbol{g}^{\dagger} \boldsymbol{g}\right)=\log _{2} e \int_{0}^{\infty} \frac{1}{z}\left(1-e^{-z \boldsymbol{g}^{\dagger} \boldsymbol{g}}\right) e^{-z} d z
$$

in which the quadratic form $\boldsymbol{g}^{\dagger} \boldsymbol{g}$ appears only at the exponent.

Therefore, from (7) and (5), the ergodic capacity (2) can be evaluated as follows

$$
\begin{aligned}
& \mathcal{C}=\log _{2} e \int_{0}^{\infty} \frac{1-\mathcal{M}(z)}{z} e^{-z} d z \\
& =\log _{2} e \int_{0}^{\infty} \frac{1}{z}\left(1-\frac{1}{\operatorname{det}\left(\boldsymbol{I}_{M}+z \boldsymbol{\Lambda}\right)} e^{-\mu^{\dagger}\left[z^{-1} \boldsymbol{I}_{M}+\boldsymbol{\Lambda}\right]^{-1} \mu}\right) e^{-z} d z
\end{aligned}
$$

which involves only one single-integral over the non-negative real line $\mathbb{R}^{+}$.

As far the evaluation of the integral in (8) is concerned, we prove in what follows that the integrand is continuos and bounded (and therefore has no singular points in the range of integration). To prove this, notice from [21, Equ. 2.6, pp.
435]) that the term $\frac{1-\mathcal{M}(z)}{z}$, with $\mathcal{M}(z)=\mathbb{E}\left[e^{-z \boldsymbol{g}^{\dagger} \boldsymbol{g}}\right]$, is related to the tail probability as follows

$$
\frac{1-\mathcal{M}(z)}{z}=\int_{0}^{\infty} e^{-z x} \operatorname{Pr}\left\{\boldsymbol{g}^{\dagger} \boldsymbol{g}>x\right\} d x .
$$

Now, owing to the fact that $0 \leq \operatorname{Pr}\left\{\boldsymbol{g}^{\dagger} \boldsymbol{g}>x\right\} \leq 1$, it can be shown by applying the Steffensen's inequality for integrals [19, Equ. 12.316] that $\forall z \in \mathbb{R}^{+}$

$$
\begin{aligned}
0 & <\frac{1-\mathcal{M}(z)}{z} \\
& =\int_{0}^{\infty} e^{-z x} \operatorname{Pr}\left\{\boldsymbol{g}^{\dagger} \boldsymbol{g}>x\right\} d x \leq \int_{0}^{\mathbb{E}\left[\boldsymbol{g}^{\dagger} \boldsymbol{g}\right]} e^{-z x} d x \\
& \leq \frac{1-e^{-z \mathbb{E}\left[\boldsymbol{g}^{\dagger} \boldsymbol{g}\right]}}{z} \leq \mathbb{E}\left[\boldsymbol{g}^{\dagger} \boldsymbol{g}\right]
\end{aligned}
$$

where we have used the fact that, for any non-negative random variable $X, \int_{0}^{\infty} \operatorname{Pr}\{X>x\} d x=\mathbb{E}[X]$.

(9) proves that the integrand in (8) is bounded. Furthermore, it can be seen that it is also continuous and possess all derivatives $\forall z \in \mathbb{R}^{+}$. Therefore, standard numerical integration packages can be used straightforwardly to compute (8)

To summarize, (8) gives the ergodic capacity of MRC over correlated Rician fading directly in terms of the covariance matrix $\Lambda$ and the mean vector $\mu$. It should be emphasized at this point that though (8) involves a single numerical integration, however it offers a huge reduction in the required computational complexity when compared to the direct method (4) which requires $M$-fold integrals. On the other hand, when comparing the computational complexity of the new expression (8) with other known methods, it is to be noticed that the ergodic capacity (8) is given directly in terms of the original covariance matrix $\Lambda$, without the need of any eigendecomposition operations. This is in contrast to most previous research on the capacity of MRC over correlated fading channels (e.g. [5], [6], [9]-[11]) which require all distinct eigenvalues of the covariance matrix with their multiplicities. It is also worth mentioning that although an expression for the pdf of the combined SNR can be obtained by the Laplace inversion of $\mathcal{M}(z)$, however this would take the form of an infinite series (Laguerre-series) and involves (in addition to the eigendecomposition of the covariance matrix) solving a large set of linear equations recursively (e.g., [9], [11], [18]).

\section{NUMERICAL EXAMPLES}

This section gives some numerical examples that demonstrate the effects of correlation on the ergodic capacity. In Fig. 1, we consider a constant correlation model in Rayleigh fading $(\kappa=0)$ with $\rho_{i j}=\operatorname{SNR} \rho \forall i \neq j$, and $\rho_{i i}=\mathrm{SNR}$, $i=1,2, . ., M$, where SNR is the average signal-to-noise ratio (SNR) per branch. Here, we plot the ergodic capacity against SNR for $M=2$ and 10 branches in cases of uncorrelated, and negatively and positively correlated branches. Fig. 1 shows that correlation (positively or negatively) decreases the ergodic capacity of MRC in Rayleigh fading. However, the loss in ergodic capacity does not exceed $7 \%$ when $M=10$. (from $11.554 \mathrm{bps} / \mathrm{Hz}$ into $10.798 \mathrm{pbs} / \mathrm{Hz}$ at $\mathrm{SNR}=25 \mathrm{~dB}$ ). We also note from Fig. 1, that negative and positive correlations result in identical capacities in case of Rayleigh fading. 


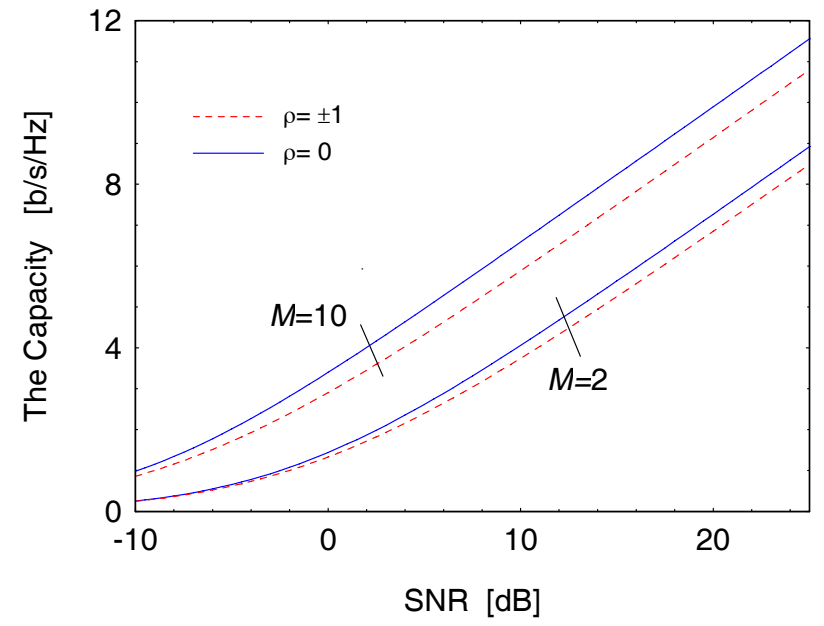

Fig. 1. The ergodic capacity against SNR $[\mathrm{dB}]$ in case of $M=2$ and 10 . $\rho=0$ and \pm 1 in case of Rayleigh fading channel $\kappa=0$.

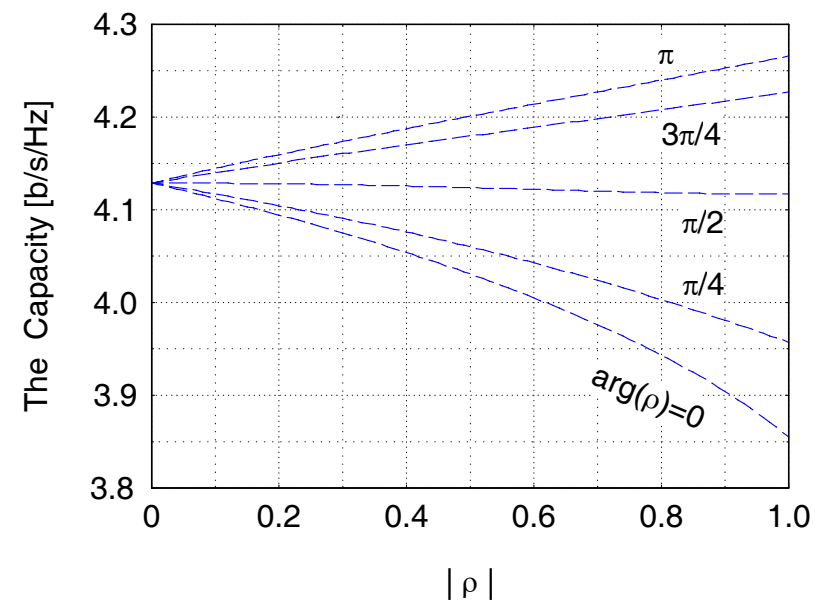

Fig. 2. The ergodic capacity against the correlation magnitude $|\rho|=1$ for several values of the correlation phase $\arg \rho=0, \frac{\pi}{4}, \frac{\pi}{2}, \frac{3 \pi}{4}, \pi . M=2$, $\kappa=1$ and $\mathrm{SNR}=10 \mathrm{~dB}$.

In order to gain insight into the effect of correlation on the ergodic capacity of MRC in Rician fading, we consider in Figs. 2-4 a dual diversity system in a Rician fading scenario of mean vector $\mu_{m}=\sqrt{\frac{\kappa}{1+\kappa} \mathrm{SNR}}, m=1,2$, and a covariance matrix

$$
\Lambda=\left[\begin{array}{ll}
\frac{\mathrm{SNR}}{1+\kappa} & \frac{\mathrm{SNR}}{1+\kappa} \rho \\
\frac{\mathrm{SNR}}{1+\kappa} \rho^{*} & \frac{\mathrm{SNR}}{1+\kappa}
\end{array}\right]
$$

with $\mathrm{SNR}=10 \mathrm{~dB}$.

In Fig. 2, we let the Rice factor $\kappa=1$, and plot the ergodic capacity against the correlation's magnitude $|\rho|$ for several correlation's phase $\arg \rho=0, \frac{\pi}{4}, \frac{\pi}{2}, \frac{3 \pi}{4}, \pi$. We observe that, in contrast to Rayleigh fading, the ergodic capacity depends on the correlation's phase. Furthermore, depending on the size of the correlation's phase, the ergodic capacity can be increased with increasing the magnitude of the correlation. Specifically, as can be seen from Fig. 2, the ergodic capacity increases with increasing $|\rho|$ when $\arg \rho>\pi / 2$.

In Fig. 3, we plot the ergodic capacity against $\arg \rho$ when $|\rho|=1$ and $\kappa=0,0.2,0.5,1,5$. We observe that when $\kappa=0$, then the phase $\arg \rho$ has no affect on the capacity. However,

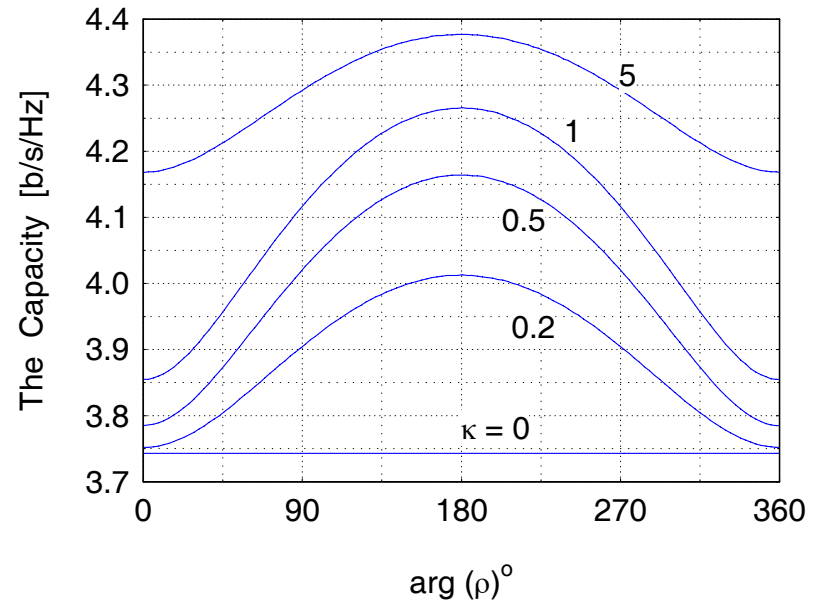

Fig. 3. The ergodic capacity against the correlation phase [degrees] $\arg \rho^{\circ}$ for fully correlated dual branches with $|\rho|=1$ and several values of Rice factor $\kappa=0,0.2,0.5,1,5 . \mathrm{SNR}=10 \mathrm{~dB}$.

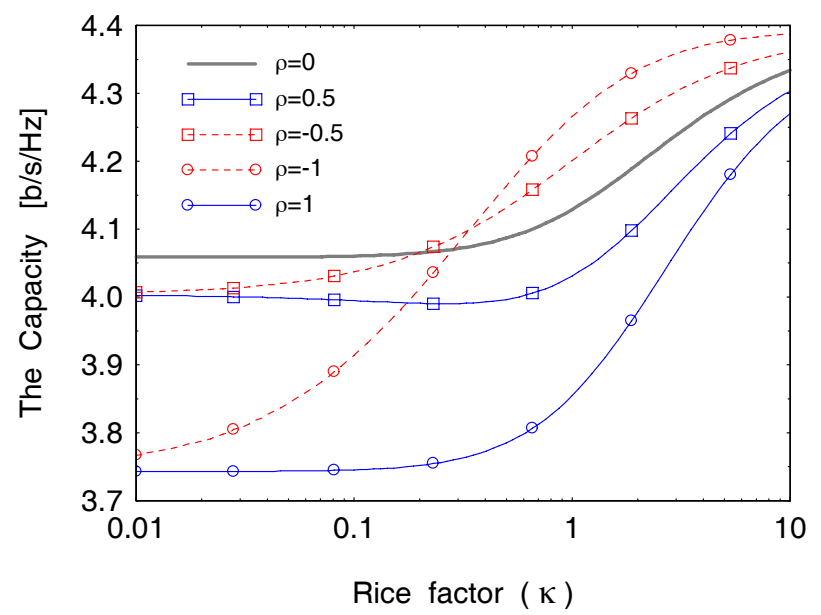

Fig. 4. The ergodic capacity against the Rice factor $\kappa . M=2, \mathrm{SNR}=10$ $\mathrm{dB}$, and for several correlation coefficients $\rho=0, \pm 0.5, \pm 1$.

when $\kappa>0$, then the ergodic capacity is maximized when $\arg \rho=\pi$ (i.e., negative correlation). In Fig. 4, we plot the ergodic capacity against $\kappa$ for different correlations. One can make the following observations from Figs. 2-4: 1) For a given correlation, increasing the Rice factor $\kappa$ leads to an increase in the ergodic capacity. 2) For any value of Rice factor $\kappa$, the capacity with positively correlated branches does not exceed that with negatively correlated branches. 3) Negatively correlated branches perform better than uncorrelated branches when $\kappa>0.4$. On the other hand, increasing the magnitude of correlation when $\kappa<0.2$ causes to decrease the ergodic capacity.

\section{SUMMARY}

A new simple expression is derived for computing the ergodic capacity of MRC with arbitrarily correlated Rician faded branches. This is used to determine the effect of correlated branches on the performance of MRC diversity. Numerical results indicate that the ergodic capacity of MRC in negatively correlated Rician fading channels can be improved beyond what would be achieved in uncorrelated channels. 


\section{APPENDIX}

In order to give a formal proof of Lemma 1, consider the following series expansion of $\ln (1+x)$ which is valid for all $x \geq 0$ [20, Eq. 4.1.25]

$$
\ln (1+x)=\sum_{n=1}^{\infty} \frac{1}{n}\left(\frac{x}{1+x}\right)^{n}, x \geq 0 .
$$

Now, using the identity ${ }^{1}$ (e.g. [19, Eqs. 8.312.2 or 3.381.4])

$$
x^{n}=\int_{0}^{\infty} \frac{s^{n-1}}{\Gamma(n)} e^{-s / x} d s, \quad n, x>0
$$

(10) becomes

$$
\begin{aligned}
\ln (1+x)= & \sum_{n=1}^{\infty} \frac{1}{n} \int_{0}^{\infty} \frac{s^{n-1}}{\Gamma(n)} e^{-s \frac{1+x}{x}} d s \\
= & \int_{0}^{\infty}\left\{\sum_{n=1}^{\infty} \frac{1}{n} \frac{s^{n-1}}{\Gamma(n)}\right\} e^{-s \frac{1+x}{x}} d s \\
& \int_{0}^{\infty}\left\{\frac{1}{s}\left(e^{s}-1\right)\right\} e^{-s \frac{1+x}{x}} d z
\end{aligned}
$$

which reduces to (6) when we substitute $s=z x$.

\section{REFERENCES}

[1] H. T. Hui, "The performance of the maximum ratio combining method in correlated Rician-fading channels for antenna-diversity signal combining," IEEE Trans. Antennas Propag., vol. 53, no. 3, pp. 958-964, Mar. 2005.

[2] E. A. Jorswieck, T. J. Oechtering, and H. Boche, "Performance analysis of combining techniques with correlated diversity," IEEE WCNC'05, pp. 849-854, Mar. 2005.

[3] H. Boche and E. A. Jorswieck, "On the ergodic capacity as a function of the correlation properties in systems with multiple transmit antennas without CSI at the transmitter," IEEE Trans. Commun., vol. 52, no. 10, pp. 1654-1657, Oct. 2004.

[4] R. Annavajjala and L. B. Milstein, "On the capacity of dual diversity combining schemes on correlated Rayleigh fading channels with unequal branch gains," IEEE WCNC'04, pp. 300-305, Mar. 2004.

[5] H. Zhang, W. Li, and T. A. Gulliver, "Capacity and error probability of orthogonal space time block codes over correlated Rayleigh and Rician channels," IEICE Trans. Fundamentals of Elec., Commun., and Comp. Science, vol. E 88-A, no. 11, pp. 3203-3213, Nov. 2005.

[6] A. Forenza, M. R. McKay, I. B. Collings, and R. W. Heath Jr., "Switching between OSTBC and spatial multiplexing with linear receivers in spatially correlated MIMO channels," in Proc. IEEE Veh. Technol. Conf. (VTC), Melbourne, Australia, May 2006.

[7] Q. T. Zhang and D. P. Liu, "A simple capacity formula for correlated diversity Rician fading channels," IEEE Commun. Lett., vol. 6, no. 1, pp. 481-483, Nov. 2002.

[8] J. Perez et al., "Tight closed-form approximation for the ergodic capacity of orthogonal STBC," IEEE Trans. Wireless Commun., vol. 6, no. 2, pp. 452-457, Feb. 2007.

[9] L. Musavian, M. Dohler, M. R. Nakhai, and A. H. Aghvami, "Closedform capacity expressions of orthogonalized correlated MIMO channels," IEEE Commun. Lett., vol. 8, no. 6, pp. 365-367, June 2004.

[10] S. Furrer and P. Cornoel, "Simple ergodic and outage capacity expressions for correlated diversity Ricean fading channels," IEEE Trans. Wireless Commun., vol. 5, no. 7, pp. 1606-1609, July 2006.

[11] R. U. Nabar, H. Bolcskei, and A. J. Paulraj, "Diversity and outage performance in space-time block coded Ricean MIMO channels," IEEE Trans. Wireless Commun., vol. 4, no. 5, pp. 2519-2532, Sept. 2005.

[12] S. Khatalin and J. P. Fonseka, "On the channel capacity in Rician and Hoyt environments with MRC diversity," IEEE Trans. Veh. Technol., vol. 55, no. 1, pp. 173-141, Jan. 2006.

${ }^{1}$ Notice that (11) is common in communication engineering. For instance, it is related to gamma probability density function, and the Laplace transform of $t^{n-1}$.
[13] M. K. Simon and M.-S. Alouini, "A unified approach to the probability of error for noncoherent and differentially coherent modulations over generalized fading channels," IEEE Trans. Commun., vol. 46, no. 12, pp. 1654-1657, Dec. 1998.

[14] V. V. Veeravalli, "On performance analysis for signaling on correlated fading channels," IEEE Trans. Commun., vol. 49, no. 11, pp. 1879-1883, Nov. 2001.

[15] M. Yao, "Impact of correlated diversity branches in Rician fading channels," in Proc. IEEE ICC'05, pp. 473-477, May 2005.

[16] M.-S. Alouini and A. Goldsmith, "A unified approach for calculating error rates of linearly modulated signals over generalized fading channels, IEEE Trans. Commun., vol. 47, no. 92, pp. 1324-1334, Sept. 1999.

[17] M. Schwarz, W. R. Bennet, and S. Stein, Communication Systems and Techniques. New York: McGraw-Hill, 1966.

[18] A. M. Mathai and S. B. Provost, Quadratic Forms in Random Variables, Theory and Applications, New York: Marcel Dekker, 1992.

[19] L. S. Gradshteyn and I. M. Ryzhik, Table of Integrals, Series, and Products, 6th ed. Academic Press, 2000.

[20] S. M. Abramowitz, Handbook of Mathematical Functions with Formulas, Graphs, and Mathematical Tables. U.S. Department of Commerce, 1972.

[21] W. Feller, An Introduction to Probability Theory and Its Applications, vol. II, 2nd ed. John Wiley \& Sons, 1971. 